\title{
A Conceptual Study on User Identification and Verification Process using Face Recognition Techniques
}

\author{
K. Krishnaprasad ${ }^{1}$ \& P. S. Aithal ${ }^{2}$ \\ ${ }^{1}$ Research Scholar, Rayalaseema University, Kurnool-518 007, Andra Pradesh, India \\ ${ }^{1,2}$ Srinivas Institute of Management Studies, Pandeshwar, Mangalore - 575 001, India \\ E-Mail: karanikrishna@gmail.com
}

Type of the Paper: Research Paper.

Type of Review: Peer Reviewed.

Indexed In: OpenAIRE.

DOI: http://dx.doi.org/10.5281/zenodo.810343.

Google Scholar Citation: IJAEML

\section{How to Cite this Paper:}

Krishnaprasad K., Aithal, P. S. (2017). A Conceptual Study on User Identification and Verification Process using Face Recognition Techniques. International Journal of Applied Engineering and Management Letters (IJAEML), 1(1), 6-17.

DOI: http://dx.doi.org/10.5281/zenodo.810343.

International Journal of Applied Engineering and Management Letters (IJAEML) A Refereed International Journal of Srinivas University, India.

(c) With Authors.

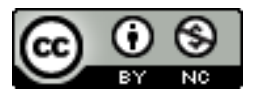

This work is licensed under a Creative Commons Attribution-Non Commercial 4.0 International License subject to proper citation to the publication source of the work.

Disclaimer: The scholarly papers as reviewed and published by the Srinivas Publications (S.P.), India are the views and opinions of their respective authors and are not the views or opinions of the S.P. The S.P. disclaims of any harm or loss caused due to the published content to any party. 


\title{
A Conceptual Study on User Identification and Verification Process using Face Recognition Techniques
}

\author{
K. Krishnaprasad ${ }^{1}$ \& P. S. Aithal ${ }^{2}$ \\ ${ }^{1}$ Research Scholar, Rayalaseema University, Kurnool-518 007, Andra Pradesh, India \\ ${ }^{2}$ Srinivas Institute of Management Studies, Pandeshwar, Mangalore - 575 001, India \\ E-Mail: karanikrishna@gmail.com
}

\begin{abstract}
The rapid growth of information and communication technology resulted in abundant data and information growth in every field with the necessity for high data security. Face recognition is one of the types of unique biometrics measure related to human characteristics, which can be used for identification or authentication purpose as individual's claimed identity. Face recognition system acquired great scope for the past few years in image processing for the security purposes including identification and verification process, due to its applications in various domains like crime detection, banking, and defense. Based on face data acquisition, face recognition techniques can be broadly classified into three types as intensity images using local binary pattern, video sequences using training videos and 3D information using 3D feature extraction. This paper discusses different face recognition algorithms and analyses it uses its advantages and disadvantages. This paper also compares and discusses how these techniques can be used for various identification and verification system in various fields and attempts to disclose state of the art of face recognition technology.
\end{abstract}

Keywords: Face recognition, Verification, Identification, Image processing.

\section{INTRODUCTION :}

Face recognition is one of the important biometrics research problems spanning across different fields like computer vision, image processing and many another disciplines of computer science. Face acts as not only fundamental physical part of human being for effective communication and interaction among people but also having enormous practical application in diverse fields like bankcard identification, access control, security monitoring, crime detection and much more. Facial Recognition is a type of biometric software application out of many types of finger scan, iris scan, retina scan, hand scan, voice scan, signature scan and keystroke scan etc, which can identify or recognize or verify a particular or specific person by analyzing and comparing face image against already stored image in the database.

An official method of categorizing/classifying faces was first proposed in [1]. The author proposed face as a profile, which is a collection of curves, finding their norm, and then classifying other profiles based on deviations from this norm. This classification is considered to be multimodal classification. Active and advanced development of algorithms, the availability of large databases of facial images, a different method for analyzing and evaluating the performance of face recognition algorithm and different test tools are the major reasons for the rapid development of face recognition system. Face recognition system starts with detection of face in jumbled or unordered scenes, proceeds further by normalizing the face image for geometrical and illuminations changes, which considers information about location and appearance of face landmarks and later identifies face using proper classification algorithm and finally process the results based on model based schema and logistic feedback [2]. Depending on the kind of images and recognition algorithms, the development of face recognition 
allows classification into three different types, namely frontal, profile and view tolerant recognition. Frontal recognition is most common and classical approach, and view tolerant algorithm usually performs recognition in more sophisticated fashion by considering different underlying subjects like physics geometry and statics. Profile schema as a standalone system has a trivial significance for identification [3]. Based on the pose invariance, face recognition system may be classified into two types as global approach and component based approach. There are several classifier approaches are available in the literature minimum distance classification used in eigenspace [4, 5], Fisher Discriminate analysis [6] and neural network [7]. Global techniques are well suited for classifying frontal view of the faces. However, they are not strong against pose changes due to highly sensitive to translation and rotation of the face image [8]. To overcome this drawback an alignment stage is added prior to classifying the face. In aligning phase, correspondence between input face image and reference face image is essential to work smoothly. The correspondence is considered for very minute points the face, which includes the center of the eye, the nostrils, or the corners of the mouth. In order to significantly distort image [9], affine transformations are carried out. To align input face with the model face, active shape models are used effectively, which is explained in [10]. Support vector machine classification was combined with semi-automatic alignment step is proposed in [11]. In reference [12] face recognition was performed by template matching independently, three different parts of the face as eye, nose, and mouth. Since the system did not include the geometrical model of the face, the classification was unconstrained. A similar approach by adding one alignment stage was proposed in [13].

Face recognition system are still faces challenges in some specific domains like illumination changes pose changes. The False rejection rate is the system fails to identify an enrollee, can be increased when enrollee wears glass, changes hair style, grow or shave a beard etc. Even though there are numerous methods have been proposed and developed to overcome these drawbacks and promised to solve these hurdles, the difficulty still remains. The matching performance in most of the automatic face recognition system is relatively poor compared to other biometric features like fingerprint and iris matching. The system works well if the condition and environmental changes are similar in the case of capturing training and test images. Generally, there are three kinds of methods that cope with variations in appearance, which are invariant features, canonical forms, and variations-modeling. The first approach seeks to utilize features that do not change with respect to variations in appearance. Even though lighting/illumination variation condition changes, that do not affect the performance in the Quotient image [14]. The second approach attempts standardize or normalize the local features or simply the variations, through image transformation and synthesizing test image as a mathematical or canonical form. Recognition or identification is done based on this canonical form. These approaches are explained in $[15,16]$. The third approach is variations-modeling, in which two important factors are considered as suitable subspace and the extent of variations in that space. In this method some parameterization of the subspaces is essential. While performing recognition, subspace closest to the test image are considered and then appropriately mapped. The identification and verification process are able to find variations or pose changes. These methods are explained in [17-20].

Even though many methods and algorithms are developed for face recognitions by valiant work of researchers, it remains still difficult problem in general. Each of the algorithm works well for some of the specific variations, which are thoroughly analyzed and studied in the research, but causes problems when other types of variations occur. For example, some methods or techniques works well for illumination changes but fails when pose or facial expression changes. For a verification system, this is not a big problem when training and test databases are captured during same conditions but are not robust enough for unconstrained, general recognition systems.

In this paper first, we explain the basic of face recognition, verification, and validation process using introductory section. A brief literature review on Face recognition studies, which includes different methods as Eigenfaces (Eigen features), neural networks, hidden Markov model, geometrical feature matching, and template matching are considered, which apply mostly to frontal faces. The third section covers basics of face recognition with face recognition process and four stages of Identification and verification process. The fourth Section narrates about applications of identification 
and verification in face recognition. Section five covers analysis of different face recognition techniques with its merits and demerits using tabular form. Section six concludes the paper.

\section{FACE RECOGNITION TECHNIQUES :}

In these section different face recognition techniques like Eigenfaces (Eigen features), neural networks, hidden Markov model, geometrical feature matching, and template matching are considered, which apply mostly to frontal faces.

\section{A. Eigenfaces}

Eigenface is one of the most common, popular and thoroughly investigated technique for face recognition. Based on the techniques and method used, this is also known as eigenpicture, eigenvector, principal component analysis and Karhunen-Loeve expansion. References [21, 22] represented the picture of the face through principal component analysis. Using a standard face picture (eigenpicture) and small collections of weights for each phase, any phase image can be approximately reconstructed. In mathematical terms, we can define Eigenfaces are eigenvectors of the covariance matrix of the set of the face images which can be having different applications in the diverse field. Every face image can be represented as linear combinations of the Eigenfaces. Usually, eigenvectors do not give $100 \%$ correct classification and are usually approximated to $95 \%$ or $85 \%$ or even 64\%., based on lighting, orientation and size variations respectively. Reference [23] confirmed that association between images of entire face is not proficient for proper recognition purpose, whereas illumination normalization [21] a more efficient method for satisfactory recognition performance in Eigenface method. References [24] considered three images, which is taken in different lighting or illumination conditions for the purpose of computing covariance matrix, which was considered as one of the new methods. Reference [25] considered eigen features analogous to face components, such as eye, nose, and mouth which are an extended work of their previous work. They modeled their work on eigeneyes, eigennose, and eigenmouth and this method is more efficient than standard eigenface method. They have considered sample and test database as FERET, consisting of 3000 person's different pose and illumination of 7562 images approximately. Reference [26] considered principal component analysis approach with the aid of ear and face recognition, and their result showed that recognition performance does not change significantly when image changes but changes when multimodal biometrics is used for identification purpose. There is much related research are available in the area of multimodal biometrics. Reference [27] uses face and fingerprint in the identification and Reference [28] used to face and voice.

\section{B. Neural Networks}

Nonlinearity in the network is one prominent reason for usage of Neural Networks. The single Layered Adaptive network called as WISARRD, in which each individual will be having one separate network structure. This is considered to be one of the first Artificial Neural Network (ANN) used for face recognition [29]. The efficiency of Neural Networks depends on the way in which neural network structure is constructed and it varies from applications to applications. For face detection, multilayer Perceptron and convolutional neural network and for face verification multi-resolution pyramid techniques are best-suited methods or techniques [30-32]. Reference [31] proposed mixed or hybrid Neural Network which is combinations of local image sampling, a Self-Organizing Map neural network and a convolutional neural Network. The SOM initially does the quantization of the image sample space and maps to topological space, which helps to reduce dimension reduction. In this process inputs which are nearby in the original sample space will be there in the output sample space also. The convolutional network extracts more features in the hierarchical set of layers and provides satisfactory invariance to basic transformations which include translation, rotation scaling, and deformation. This method takes for training as long as 4 hours and for classification only 0.5 seconds.

\section{Graph Matching}

Graph Matching is another technique to face recognition. Reference [33] presented a dynamic link structure for deformation resistant object recognition which used elastic group matching to find the closest or closely related stored graph. Dynamic link architecture is similar to artificial neural networks with some extended advanced features. Sparse graphs contain memorized objects. Sparse 
graph vertices are labeled in terms of a local power spectrum with a multi-resolution description and sparse graph edges are labeled with geometrical distance vectors. Object recognition can be invented as elastic graph matching which is made into work with the aid of stochastic optimization of matching cost function.

Reference [34] extended the technique by making further study and compared and later matched human faces against 112 neutral frontal view faces. Using the depth characteristics of rotation and facial expression changes, probe images are distorted or became vague. They have reported a significant recognition rate of $86.5 \%$ and $66.4 \%$ for matching tests of 111 and 112 faces of 15 -degree rotation and 30-degree rotation respectively to a gallery of 112 neutral faces.

\section{Hidden Markov Model (HMMs)}

Hidden Markov Model was successfully applied for speech application with the aid of non stationary vector time series. References [35] used this technique human face recognition. Faces were spontaneously divided into regions such as eyes, nose, mouth, etc., which can be studied with the aid of HMMs model. HMMS requires image samples in one-dimensional observation sequences, the two dimensional images are to be converted into either ID temporal or ID spatial sequences. In [36], with the help of band sampling technique, spatial observation sequences are extracted from the face image. One-dimensional vector series of pixel observation were used in order to represent each face image. Each observation vector is made by a block of lines referred as L lines. M Lines overlap between successive observations. An unidentified test image is considered an initial sample for this observation sequence and later it is matched and tested against every HMMs in the face database. The match with highest or most scoring is considered to be the relevant model and that best reveals the identity of the test face.

\section{E. Geometrical Feature Matching}

Geometrical Feature Matching techniques are based on a set of geometrical features of face images, which includes eye color, skin color, and shape of the nose etc. The face recognition is possible even small or vague resolution as 8x6 pixels when single facial features are heavily analyzed and studied and this also reveals one factor that in order to identify or verify face overall geometrical face features of the face is also sufficient [37]. Usually, an overall description of the face is described using a vector. This vector represents position and size of the main facial features such as eye, eyebrows, mouth, nose, ear, teeth and other parts of the face and the shape of face outline. One of the important and revolutionary works on automated face recognition by using geometric feature was studied by [38] in 1973. They took a database of 20 people using model and test image for each person and their system was able to give $75 \%$ recognition rate and that was considered to be one of the best performance at that time. References [39, 40] showed that features extracted through manually could also be used for face recognition with satisfactory results. Nose width and length, mouth position, and chin shape are some of the features of the face, which are automatically extracted and considered as geometrical features [41]. 35 features are extracted from 35-dimensional vectors and face recognition was done with a Bayes Classifier.

\section{F. Template Matching}

In Template Matching, sometimes a single template represents the whole face. A two- dimensional array of intensity values are compared using the suitable metric like Euclidean distance. Twodimensional arrays are generated from the test image. The above mentioned is a simple version of template matching. In literature, there are other more sophisticated methods of Template Matching on face recognition. In complex face recognition techniques more than one template are considered for individual face, which is taken from the different viewpoints. A set of multiple distinct smaller templates is prepared from the only single viewpoint of face image [42, 43]. In the face image, there will be gray levels, when we consider gray levels of the original image that also processed properly before matching [44]. In [42] Bruneli and Poggio automatically selected more than one feature templates of individual's face in order to recognize the face for identification/verification purpose, which are eyes, nose, mouth, and whole face. They used a database of 47 individuals as training database, which contains 188 images of the face and using this database, they compared the performance of their geometrical matching algorithm and Template Matching algorithm. They found 
that Template Matching was superior to geometrical matching, giving $100 \%$ and $90 \%$ respectively recognition rate.

\section{FUNDAMENTALS OF FACE RECOGNITION :}

As shown in Figure-1, the major components of face recognition system include mainly three components as Enrolment/Registration module, Database, and Identification or Verification module. The Enrolment/ Registration module is an automated mechanism that scans or captures a digital image of a face from a still image or lives video. This image includes faces and non-faces also in big size. Initially, face image contains entire face image or picture. This image contains noises. One of the most common types of noise is light illumination. Other types of noises are pose variations, occlusion and image orientation [45-46]. From this image, face part is captured and processed through features extraction. These features are stored as pattern or templates. The database is responsible for storage of the image in compressed, processed and small sized format or as a template, which can be accessed at any time using database operations. The third module- identification/verification module or interface resides in application system which mainly compares the extracted features of the image with already stored image/template in the database. Finally, based on the matching score/ranks of image, a decision is made for either to accept or reject the face, considered for identification and verification recognition purpose. In identification and Verification process new sample image again has to pre-process in order to eliminate noises and to create a template.

The identification and verification are the two types of comparisons made by any type of recognition algorithms. In identification, process system compares unknown face image, which is given as input for the system with all stored faces in the database and also returns the best matching or the best ranked images. In the verification process system compares the given individual input image with whom that person or individual claims to be and returns a Boolean value as yes or no. In identification process, one-to-many comparisons are done, whereas in verification one-to-one comparisons are carried out.

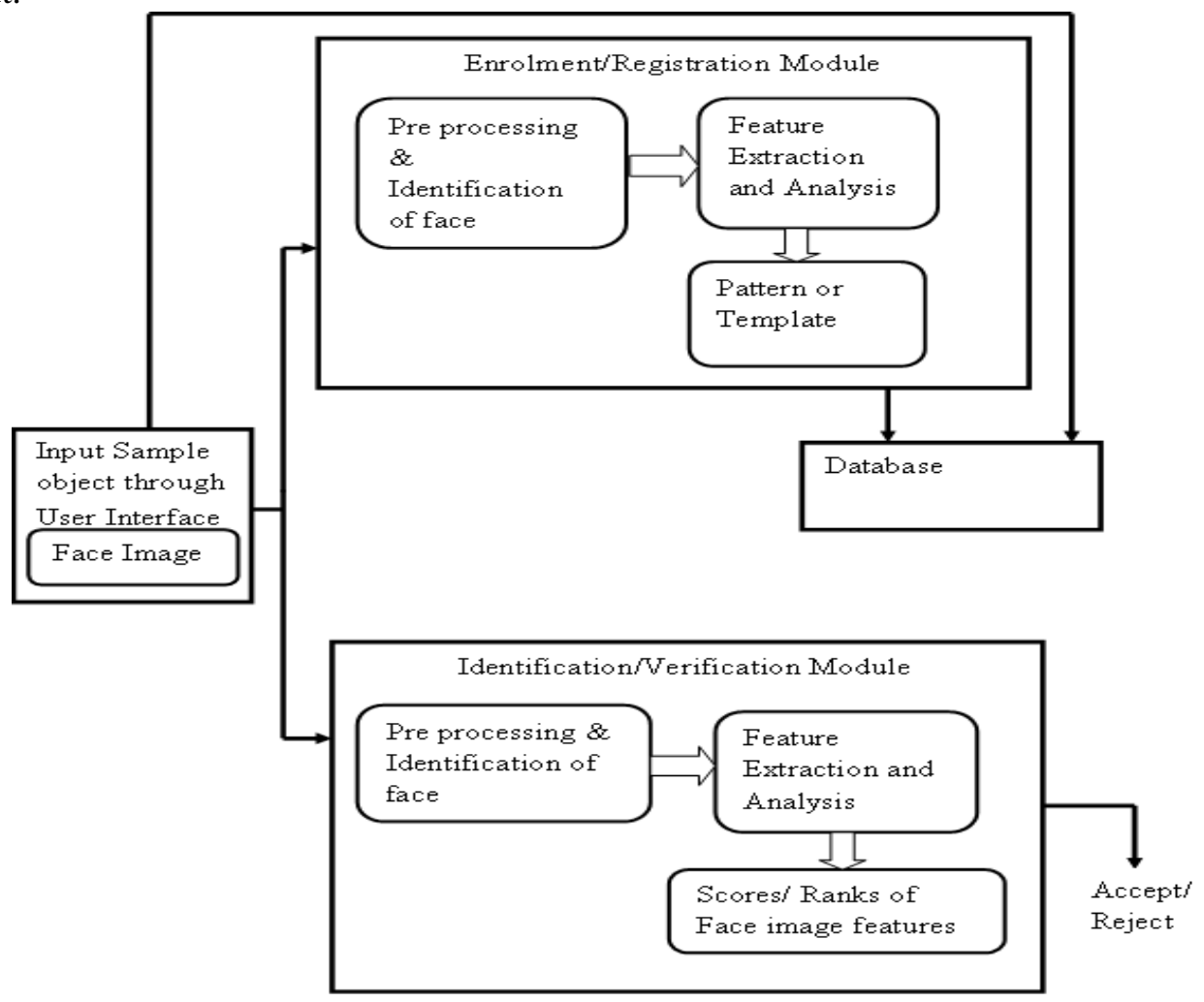

Fig. 1: Face Recognition Process 


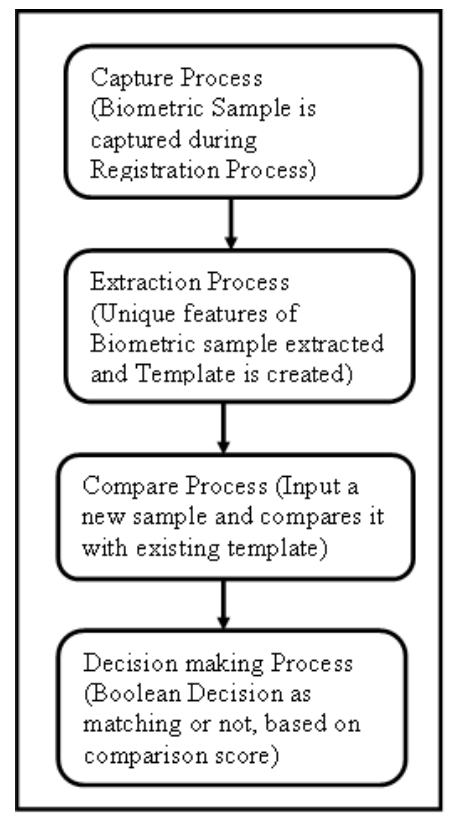

Fig. 2: Identification and Verification's Four Major Process

As shown in figure-2, all biometric based identification or authentication system works based on major four processes;

(1) Capture - A physical sample or one of the biometric image instances is captured by the system during the registration process, which is true even in the case of identification or verification stage. In capture process biometric can be finger scan, iris scan, retina scan, hand scan, voice scan, signature scan and keystroke scan etc.

(2) Extraction - Unique characteristics or features of the instance are extracted or retrieved and a template or pattern is created. Features can include unique features of the face components such as eye, nose, and mouth.

(3) Compare - System takes or considers a new sample as an input and compares it with an already stored pattern or template. Different algorithms give varying score levels based on input parameters.

(4) Decision Making - The system makes a Boolean decision as matching or not matching based on the similarity between feature extracted from the new instance and already stored template.

\section{APPLICATIONS OF IDENTIFICATION AND VERIFICATION PROCESS USING FACE RECOGNITION :}

Face recognition system has got different applications in the diverse field, which includes security, access control, military, investigation department, forensic department etc. Following are the different application of verification and Identification using Face recognition.

- Face image can be effectively used in organizations in order to verify the employee identity at the entrance of the organization as Entry control system.

- Face image can be used for attendance purpose in institutions, organizations or offices using verification process.

- The Face image is vital in identifying the criminals using identification process.

- Verification and Identification can be efficiently used in country boarders to differentiate between army officials and unknown persons.

- Verification and Identification is helpful in supermarket or malls to view suspicious, malicious transactions.

- Verification and Identification can be used in face database investigations purpose, which includes benefit recipients, missing children, immigrants and police bookings

- Verification system can be used in Electoral registration, e-commerce, banking, identifying newborns, national ID and passports. 
- Central Surveillance System can use Face image for identification purpose with low user satisfaction level.

- Verification and Identification can be used in forensic security.

\section{ANALYSIS OF FACE RECOGNITION TECHNIQUES :}

In this section, different face recognition techniques, eigenfaces, neural networks, hidden Markov model, graph matching geometrical feature matching, and template matching are analyzed using its Advantages and Disadvantages [47-52].

Table 1: Advantages and disadvantages of face recognition Techniques

\begin{tabular}{|c|c|c|}
\hline Techniques & Advantages & Disadvantages \\
\hline Eigen faces & $\begin{array}{l}\text { 1. Raw intensity data are used for } \\
\text { recognition process. } \\
\text { 2. Knowledge of geometric or } \\
\text { mathematical details of face is not } \\
\text { required. } \\
\text { 3. Compression is achieved by low } \\
\text { dimensional sub spaces. } \\
\text { 4. Recognition process simpler and } \\
\text { efficient. } \\
\text { 5. Basic step of face recognition. }\end{array}$ & $\begin{array}{l}\text { 1. Learning Process is slow or time } \\
\text { consuming. } \\
\text { 2. Number of face classes should be more } \\
\text { than number of dimensions of the face } \\
\text { space. } \\
\text { 3. Overlapping of class increases when } \\
\text { more same face space represents more face } \\
\text { classes. } \\
\text { 4. Appearance based technique. } \\
\text { 5. Projection of un-known image requires } \\
\text { pixel by pixel multiplication of input image } \\
\text { 6. Sensitive to lighting conditions. }\end{array}$ \\
\hline $\begin{array}{l}\text { Neural } \\
\text { Network }\end{array}$ & $\begin{array}{l}\text { 1. Basic Perceptron Neural } \\
\text { Network (BPNN) provides } \\
\text { provision to change weight in feed } \\
\text { forward network by changing } \\
\text { activation function makes easier for } \\
\text { learn training set in input and } \\
\text { output data sets. } \\
\text { 2. Gradient descent method } \\
\text { minimizes total squared error of the } \\
\text { output computed. } \\
\text { 3. Non Linear face image can be } \\
\text { recognized easily by combining } \\
\text { PCA and BPNN. } \\
\text { 4. SOM's quantization of the image } \\
\text { sample space and mapping to } \\
\text { topological space helps to reduce } \\
\text { dimension reduction. } \\
\text { 5. RBF learns faster compare to } \\
\text { MLP. }\end{array}$ & $\begin{array}{l}\text { 1. Its slow technique. } \\
\text { 2. Training process takes more time. } \\
\text { RBF complexity increase because of } 3 \\
\text { layers as input, hidden and output. } \\
\text { 3. Requires knowledge of Data mining and } \\
\text { mathematical functions. }\end{array}$ \\
\hline $\begin{array}{l}\text { Graph } \\
\text { Matching }\end{array}$ & $\begin{array}{l}\text { 1. Sparse graphs contain } \\
\text { memorized objects } \\
\text { 2. Elastic Bunch Graph Matching } \\
\text { (EBGM) exhibits robustness under } \\
\text { illumination variations. } \\
\text { 3. Good for offline applications } \\
\text { where training images are scarce. } \\
\text { 4. Recognizes face with feature } \\
\text { changes. }\end{array}$ & $\begin{array}{l}\text { 1. High computationally complexity makes } \\
\text { it inappropriate for real time applications. } \\
\text { 2. Highly Sensitive for lighting conditions. } \\
\text { 3. High Memory Usage. } \\
\text { 4. Requires mathematical complex } \\
\text { calculation. }\end{array}$ \\
\hline $\begin{array}{l}\text { Hidden } \\
\text { Markov }\end{array}$ & $\begin{array}{l}\text { 1. Better performance rate. } \\
\text { 2. Better recognition rate. }\end{array}$ & $\begin{array}{l}\text { 1. Algorithm is expensive in terms of } \\
\text { memory and compute time. }\end{array}$ \\
\hline
\end{tabular}




\begin{tabular}{|c|c|c|}
\hline Model & $\begin{array}{l}\text { 3. The Faces were spontaneously } \\
\text { divided into regions such as eyes, } \\
\text { nose, mouth, etc., which is easy to } \\
\text { understand. } \\
\text { 4. The Promising method works } \\
\text { well images with variations in } \\
\text { lighting, facial expression and } \\
\text { orientation. }\end{array}$ & $\begin{array}{l}\text { 2. Trained on a set of seed sequences } \\
\text { requires larger seed. } \\
\text { 3. Training involves repeated iterations. } \\
\text { 4. Requires more mathematical } \\
\text { computations. }\end{array}$ \\
\hline $\begin{array}{l}\text { Geometrical } \\
\text { Feature } \\
\text { Matching }\end{array}$ & $\begin{array}{l}\text { 1. Small or vague resolution as } 8 \times 6 \\
\text { pixels, features are heavily } \\
\text { analyzed and studied. } \\
\text { 2. Localization and dense set of } \\
\text { facial points makes easy to } \\
\text { understand. } \\
\text { 3. Geometrical Feature Matching } \\
\text { includes eye color, skin color and } \\
\text { shape of the nose etc., which are } \\
\text { easy for processing and understand. } \\
\text { 4. Image without disturbance is } \\
\text { recognized fast. }\end{array}$ & $\begin{array}{l}\text { 1. Dimensionality reduction becomes s } \\
\text { complex task. } \\
\text { 2. Involves heavy mathematical } \\
\text { calculations. } \\
\text { 3. Because of appearance based so first } \\
\text { learning takes more time. } \\
\text { 4. Overall description of the face is } \\
\text { described for better recognition rate. } \\
\text { 5. Image disturbance reduces efficiency. }\end{array}$ \\
\hline $\begin{array}{l}\text { Template } \\
\text { Matching }\end{array}$ & $\begin{array}{l}\text { 1. Entire face can be represented as } \\
\text { template. } \\
\text { 2. The techniques can be easily } \\
\text { performed in grey images or edge } \\
\text { images. } \\
\text { 3. High correlation and low } \\
\text { correlation of the face image can be } \\
\text { distinguishable using template } \\
\text { matching. } \\
\text { 4. Simple technique for image } \\
\text { processing. }\end{array}$ & $\begin{array}{l}\text { 1. Complex recognition requires more than } \\
\text { one template. } \\
\text { 2. Vulnerabilities and threats are more for } \\
\text { template. } \\
\text { 3. Different viewpoint of face image are } \\
\text { required for effective recognition. }\end{array}$ \\
\hline
\end{tabular}

\section{CONCLUSION :}

Face recognition is emerging field in image and signal processing which has got applications in diverse fields like entry control system, access control system, security system, forensic lab, defense security, surveillance system, electoral registration, e-commerce, and banking, identifying newborns, national ID, and passports. In this paper, we made a brief survey of different face recognition techniques or algorithms, which are Eigenfaces (Eigen features), neural networks, hidden Markov model, geometrical feature matching, and template matching. Also, we discussed basic of face recognition process which identifies three components as Enrolment/Registration module, Database, and Identification or Verification module.

The identification and verification system consists of four processes as capture process, extraction process, compare process and decision-making process. We have explained different applications of verification and identification of face recognition system in diverse fields. Finally, different face recognition techniques with its advantages and disadvantages are discussed using tabular form. This paper truly attempts to disclose state of the art of face recognition technology.

\section{REFERENCES :}

[1] Francis Galton. (1998 June). Personal identification and description. In Nature, 173-177.

[2] Chellappa, Rama, Charles L. Wilson, and Saad Sirohey. (1995). Human and machine recognition of faces: A survey. Proceedings of the IEEE, 83(5), 705-741. 
[3] T. Fromherz, P. Stucki, M. Bichsel. (1997). A survey of face recognition. MML Technical Report, No 97.01, Dept. of Computer Science, University of Zurich, Zurich.

[4] Sirovich, L., \& Kirby, M. (1987). Low-dimensional procedure for the characterization of human faces. Josa a, 4(3), 519-524.

[5] Turk, M. A., \& Pentland, A. P. (1991, June). Face recognition using eigenfaces. In Proc, IEEE Conference on Computer Vision and Pattern Recognition.586-591.

[6] Belhumeur, P. N., Hespanha, J. P., \& Kriegman, D. J. (1997). Eigenfaces vs. fisherfaces: Recognition using class specific linear projection. IEEE Transactions on pattern analysis and machine intelligence, 19(7), 711-720.

[7]Fleming, M. K., \& Cottrell, G. W. (1990, June). Categorization of faces using unsupervised feature extraction. In Proc. IEEE IJCNN International Joint Conference on Neural Networks, 65-70.

[8] Ham, J., \& Lee, D. D. (2007). Separating pose and expression in face images: a manifold learning approach. Neural Information Processing-Letters and Reviews, 11(4), 91-100.

[9] Moghaddam, B., Wahid, W., \& Pentland, A. (1998, April). Beyond eigenfaces: Probabilistic matching for face recognition. In Proc. IEEE International Conference on Automatic Face and Gesture Recognition, 30-35.

[10] Lanitis, A., Taylor, C. J., \& Cootes, T. F. (1997). Automatic interpretation and coding of face images using flexible models. IEEE International Conference on Transactions on Pattern Analysis and machine intelligence, 19(7), 743-756.

[11] Jonsson, K., Matas, J., Kittler, J., \& Li, Y. P. (2000). Learning support vectors for face verification and recognition. In Proc. IEEE International Conference on Automatic Face and Gesture Recognition, 208-213.

[12] Brunelli, R., \& Poggio, T. (1993). Face recognition: Features versus templates. IEEE International Conference on transactions on pattern analysis and machine intelligence, 15(10), 1042-1052.

[13] Beymer, D. J. (1994, June). Face recognition under varying pose. In Pro., IEEE International Conference on Computer Vision and Pattern Recognition, pp. 756-761.

[14] Riklin-Raviv, T., \& Shashua, A. (1999). The quotient image: class based recognition and synthesis under varying illumination conditions. In Proc. IEEE International Conference on Computer Vision and Pattern Recognition, 2, 566-571.

[15] Zhao, W., \& Chellappa, R. (1999). Robust face recognition using symmetric shape-from-shading. Computer Vision Laboratory, Center for Automation Research, University of Maryland.

[16] Zheng, L., \& Wu, J. K. (2000). A new model-based lighting normalization algorithm and its application in face recognition (Doctoral dissertation, Master's thesis, National University of Singapore).

[17] Wiskott, L., Fellous, J. M., Kuiger, N., \& Von Der Malsburg, C. (1997). Face recognition by elastic bunch graph matching. IEEE Transactions on pattern analysis and machine intelligence, 19(7), 775-779.

[18] Zheng, L., \& Wu, J. K. (2000). A new model-based lighting normalization algorithm and its application in face recognition (Doctoral dissertation, Master's thesis, National University of Singapore).

[19] Edwards, G. J., Cootes, T. F., \& Taylor, C. J. (1998, June). Face recognition using active appearance models. In European conference on computer vision (pp. 581-595). Springer Berlin Heidelberg. 
[20] Georghiades, A. S., Belhumeur, P. N., \& Kriegman, D. J. (2000). From few to many: Generative models for recognition under variable pose and illumination. In Proc. IEEE International Conference on Automatic Face and Gesture Recognition, 277-284.

[21] Kirby, M., \& Sirovich, L. (1990). Application of the Karhunen-Loeve procedure for the characterization of human faces. IEEE Transactions on Pattern analysis and Machine intelligence, 12(1), 103-108.

[22] Turk, M., \& Pentland, A. (1991). Eigenfaces for recognition. Journal of cognitive neuroscience, 3(1), 71-86.

[23] Grudin, M. A. (1997). A compact multi-level model for the recognition of facial images Doctoral dissertation, Liverpool John Moores University.

[24] Zhao, L., \& Yang, Y. H. (1999). Theoretical analysis of illumination in PCA-based vision systems. Pattern recognition, 32(4), 547-564.

[25] Pentland, A., Moghaddam, B., \& Starner, T. (1994, June). View-based and modular eigenspaces for face recognition. In CVPR (Vol. 94, pp. 84-91).

[26] K. Chang, K.W. Bowyer and S. Sarkar (2003). Comarison and combination of ear and face images in appearance-based biometrics. IEEE Transaction On Pattern analysis and machine intelligence, 25(9), 1160-1165.

[27] Hong, L., \& Jain, A. (1998). Integrating faces and fingerprints for personal identification. IEEE transactions on pattern analysis and machine intelligence, 20(12), 1295-1307.

[28] Verlinde, P., Matre, G., \& Mayoraz, E. (1998, July). Decision fusion using a multilinear classifier. In Proceedings of International Conferences on Multisource-Multisensor Information Fusion ,1, 47-53.

[29] Stonham, T. J. (1986). Practical face recognition and verification with WISARD. In Aspects of face processing (pp. 426-441). Springer Netherlands.

[30] Sung, K., \& Poggio, T. (1995). Learning human face detection in cluttered scenes. In Computer Analysis of Images and Patterns (pp. 432-439). Springer Berlin/Heidelberg.

[31] Lawrence, S., Giles, C. L., Tsoi, A. C., \& Back, A. D. (1997). Face recognition: A convolutional neural-network approach. IEEE transactions on neural networks, 8(1), 98-113.

[32] Weng, J. J., Ahuja, N., \& Huang, T. S. (1993, May). Learning recognition and segmentation of 3d objects from 2-d images. In Computer Vision, 1993. Proceedings., Fourth International Conference on (pp. 121-128). IEEE.

[33] Lades, M., Vorbruggen, J. C., Buhmann, J., Lange, J., von der Malsburg, C., Wurtz, R. P., \& Konen, W. (1993). Distortion invariant object recognition in the dynamic link architecture. IEEE Transactions on computers, 42(3), 300-311.

[34] Wiskott, L., \& Von Der Malsburg, C. (1996). Recognizing faces by dynamic link matching. Neuroimage, 4(3), 514-518.

[35] Samaria, F., \& Fallside, F. (1993). Face identification and feature extraction using hidden markov models. Olivetti Research Limited.

[36] Samaria, F. S., \& Harter, A. C. (1994, December). Parameterisation of a stochastic model for human face identification. In Applications of Computer Vision. Proceedings of the Second IEEE Workshop,138-142.

[37] Tamura, S., Kawai, H., \& Mitsumoto, H. (1996). Male/female identification from $8 \times 6$ very low resolution face images by neural network. Pattern recognition, 29(2), 331-335. 
[38] Kanade, T. (1973). Picture processing system by computer complex and recognition of human faces. Doctoral dissertation, Kyoto University, 3952, 83-97.

[39] Goldstein, A. J., Harmon, L. D., \& Lesk, A. B. (1971). Identification of human faces. Proceedings of the IEEE, 59(5), 748-760.

[40] Kaya, Y., \& Kobayashi, K. (1972). A basic study on human face recognition. Frontiers of pattern recognition, 265-289.

[41] Brunelli, R., \& Poggio, T. (1993). Face Recognition: Features versus templates. IEEE transactions on pattern analysis and machine intelligence, 15(10), 1042-1052.

[42] Brunelli, R., \& Poggio, T. (1993). Face Recognition: Features versus templates. IEEE transactions on pattern analysis and machine intelligence, 15(10), 1042-1052.

[43] Baron, R. J. (1981). Mechanisms of human facial recognition. International Journal of ManMachine Studies, 15(2), 137-178.

[44] Bichsel, M. (1991). Strategies of robust object recognition for the automatic identification of human faces (Doctoral dissertation).

[45]Anila, S., \& Devarajan, N. (2012). Preprocessing technique for face recognition applications under varying illumination conditions. Global Journal of Computer Science and Technology, 12(11-F).

[46] Adini, Y., Moses, Y., \& Ullman, S. (1997). Face recognition: The problem of compensating for changes in illumination direction. IEEE Transactions on pattern analysis and machine intelligence, 19(7), 721-732.

[47] Krishna Prasad, K., \& Aithal, P. S. (2015). Massive Growth of Banking Technology with the Aid of 5G Technologies. International Journal of Management, IT and Engineering, 5(7), 616-627.

[48] Krishna Prasad, K., \& Aithal, P. S. (2016). The Growth of 4G Technologies in India-Challenges and Opportunities. International Journal of Management, IT and Engineering, 6(1), 543-351.

[49] Krishna Prasad, K., \& Aithal, P. S. (2015). Mobile system for Customized and Ubiquitous Learning by 4G/5G.International Journal of Management, IT and Engineering, 5(7), 63-71.

[50] Krishna Prasad, K., \& Aithal, P. S. (2016). Changing Perspectives of Mobile Information Communication Technologies towards Customized and Secured Services through 5G \& 6G. International Journal of Engineering Research and Modern Education (IJERME), 1(2), 210-224.

[51] Krishna Prasad, K., \& Aithal, P. S. (2016). An Online Comparative Study on 4G Technologies Service Providers in India. International Journal of Advanced Trends in Engineering and Technology (IJATET). 1(1), 96-101.

[52] Krishna Prasad, K. \& Aithal, P. S. (2017). A Study on Enhancing Mobile Banking Services using Location based Authentication. International Journal of Management, Technology, and Social Sciences (IJMTS), 1(1), 48-60. DOI: http://doi.org/10.5281/zenodo.583230 